\title{
EINE ERWEITERUNG DES HARTOGSSCHEN STETIGKEITSSATZES
}

\author{
WOLFGANG TUTSCHKE
}

Der bekannte Hartogssche Satz, nach dem eine Funktion von $n$ komplexen Variablen stetig ist, wenn sie von jeder einzelnen komplexen. Variablen holomorph abhängt, läßt sich in der Sprechweise der Theorie partieller komplexer Differentialgleichungen wie folgt formulieren:

Ist $f$ (fast überall in jeder $z_{j}$-Ebene) Lösung (im Sobolevschen Sinn) des Differentialgleichungssystems $\left(z_{j}=x_{j}+i y_{j}\right)$

$$
\frac{\partial f}{\partial z_{j}^{*}}=\frac{1}{2}\left(\frac{\partial f}{\partial x_{j}}+i \frac{\partial f}{\partial y_{j}}\right)=0, \quad j=1, \ldots, n,
$$

und ist $f$ eine stetige Funktion jeder einzelnen komplexen Variablen $z_{f}$, so ist $f$ auch als Funktion aller Variablen stetig ${ }^{1}$ ).

Bei einer solchen Betrachtungsweise kann man die Stetigkeit von $f$ als Folgerung des identischen Verschwindens der partiellen Ableitungen bezüglich $z_{j}^{*}$ ansehen. In dieser Arbeit soll nun gezeigt werden, daß die Stetigkeit bereits aus einer schwächeren Eigenschaft dieser partiellen Ableitungen folgt.

Von der komplexwertigen Funktion $f$ werde vorausgesetzt, daß alle partiellen komplexen Ableitungen $\partial f / \partial z_{j}^{*}$ (im Sobolevschen Sinn) existieren. Dann sei ${ }^{2}$ )

$$
g_{j}= \begin{cases}\frac{1}{f} \frac{\partial f}{\partial z_{j}^{*}}, & \text { falls } f \neq 0, \\ 0, & \text { falls } f=0 .\end{cases}
$$

\footnotetext{
${ }^{1}$ Auf die zweite Voraussetzung kann nicht verzichtet werden, wie das folgende Beispiel zeigt: Ist $f(z)=0$ für $z=0$ und $f(z)=1$ für $z \neq 0$, so ist $\partial f / \partial z^{*}=0$ fast überall, jedoch ist $f$ nicht eine stetige Funktion von $z$.

${ }^{2}$ Dieser Ansatz verallgemeinert die Definition der Hilfsfunktion $g=A+B f^{*} / f$ für $f \neq 0$ im Fall von Lösungen $w=f$ der Vekuaschen Differentialgleichung $\partial w / \partial z^{*}=A w+B w^{*} \quad$ (vgl. I. N. Vekua [9]-[10]). Die hier verwendete $n$-dimensionale Variante dieses Ansatzes wurde auch schon in $[7]-[8]$ verwendet.
} 
Hierbei sei bemerkt, daß (auch wenn im folgenden mit dem LebesgueIntegral gearbeitet wird) eine Abänderung der $g_{j}$ auf einer Menge vom Maß Null nicht zulässig sein soll ${ }^{3}$ ). Die (Sobolev-)Ableitungen von $g_{j_{1}}$ bezüglich $z_{j_{2}}, \ldots, z_{j_{\lambda}}$ werden (im Fall ihrer Existenz) mit $g_{j_{1} j_{2} \cdots j_{\lambda}}$ bezeichnet. Sind alle $j_{1}, \ldots, j_{2}$ voneinander verschieden, so werden die von ihnen verschiedenen natürlichen Zahlen zwischen 1 und $n$ mit $k_{1}, \ldots, k_{n-\lambda}$ bezeichnet. Dann gilt für (in offenen Mengen definierte) Funktionen $f$ von $n$ komplexen Variablen der folgende

$\mathrm{S}$ atz. Die Funktion $f$ ist stetig als Funktion aller Variablen, falls sie den folgenden Voraussetzungen genügt:

$1^{\mathrm{O}}$. Als Funktion jeder einzelnen komplexen Variablen ist $f$ stetig.

$2^{\mathrm{O}}$. Für alle $\lambda, 1 \leqq \lambda \leqq n$, und für alle voneinander verschiedenen ganzen Zahlen $j_{1}, \ldots, j_{\lambda}$ zwischen 1 und $n$ existieren die $g_{j_{1} \cdots j_{\lambda}}$, wobei die $\left|g_{j_{1} \cdots j_{\lambda}}\right|$ lokal beschränkt seien $\left.{ }^{4}\right)$ und außerdem im Lebesgueschen Sinn integrierbare Funktionen von $z_{j_{1}}, \ldots, z_{j_{\lambda}}$ und für jedes $k$ auch von $z_{j_{1}}, \ldots, z_{j_{\lambda}}, z_{k} \operatorname{sind}$.

$3^{\mathrm{O}}$. Ist $i_{1}, \ldots, i_{\lambda}$ eine Permutation von $j_{1}, \ldots, j_{\lambda}$, so sei $g_{i_{1} \cdots i_{\lambda}}=$ $\left.g_{j_{1} \cdots j_{\lambda}}{ }^{5}\right)$.

$4^{\mathrm{O}}$. Die $g_{j_{1} \cdots j_{\lambda}}$ seien für $1 \leqq \lambda \leqq n-1$ stetige Funktionen von $z_{k_{1}}, \ldots, z_{k_{n-\lambda}}$

$5^{0}$. In allen Punkten, in denen $f=0$ ist, sind auch alle $\partial f\left(\partial z_{j}^{*}=0{ }^{6}\right)$.

3 Das entspricht der Tatsache, daß bei einer solchen Abänderung die stetige Abhängigkeit auch von einzelnen Variablen verlorengeht. Übrigens erreicht man im folgenden größere Allgemeinheit, wenn die $g_{j}$ im Fall $f=0$ auf andere geeignete Weise definiert werden (vgl. Bemerkung 5)).

${ }^{4}$ Die Beschränktheit wird nur vorausgesetzt, um Abschätzungen der Form $(\zeta=\xi+i \eta)$

$$
\left|\iint \frac{g(\zeta)}{\zeta-z} d \xi d \eta\right| \leqq \sup |g| \iint \frac{1}{|\zeta-z|} d \xi d \eta
$$

zu ermöglichen. Anstelle der Beschränktheit kann aber auch $L^{p}$-Zugehörigkeit mit $p>2$ vorausgesetzt werden, da $(1 / p+1 / q=1)$

$$
\iint \frac{g(\zeta)}{\zeta-z} d \xi d \eta \mid \leqq\left(\iint|g(\zeta)|^{p} d \xi d \eta\right)^{1 / p}\left(\iint \frac{1}{|\zeta-z|^{q}} d \xi d \eta\right)^{1 / q}
$$

ist (vgl. I. N. Vekua [10]).

5 Falls die höheren Ableitungen von $f$ selbst existieren, kann man diese Aussage durch Verwendung der Quotientenregel herleiten (dazu muß außerdem verwendet werden, daß Ableitungen höherer Ordnung von der Reihenfolge der Differentiationen unabhängig sind).

${ }^{6}$ Funktionen mit dieser Eigenschaft könnte man in Verallgemeinerung eines von L. Bers [2] geprägten Begriffes approximativ analytisch nennen (bezüglich anderer Definitionen dieses Begriffes bei mehreren komplexen Variablen vgl. [8] und H. Begehr [1]). 
Im klassischen Fall, wo $f$ eine holomorphe Funktion jeder einzelnen komplexen Variablen $z_{j}$ ist, verschwinden alle $\partial f / \partial z_{j}^{*}$ identisch (nach den Cauchy-Riemannschen Differentialgleichungen), so daß insbesondere zunächst $5^{\mathrm{O}}$ erfüllt ist. Aber auch die $g_{j}$ und alle übrigen $g_{j_{1}} \cdots j_{\lambda}$ verschwinden identisch, so daß die Voraussetzungen $2^{\mathrm{O}}-4^{\mathrm{O}}$ trivialerweise erfüllt sind. Da die gewöhnliche komplexe Differenzierbarkeit nach $z_{j}$ die stetige Abhängigkeit von $z_{j}$ impliziert, ist auch $1^{0}$ erfüllt. Damit zeigt sich, daß der klassische Hartogssche Stetigkeitssatz als Spezialfall in dem behaupteten Satz enthalten ist.

Die behauptete Verallgemeinerung des Hartogsschen Satzes wird durch Zurückführung auf den klassischen Fall bewiesen. Diese Zurückführung wird durch Lösung der inhomogenen Cauchy - Riemannschen Differentialgleichung in $n$ komplexen Variablen realisiert. Dadurch kann man aus der gegebenen Funktion $f$ einen in allen $n$ komplexen Variablen holomorphen Faktor herausziehen. Dieser ist nach dem klassischen Hartogsschen Satz stetig. Vom zweiten Faktor der beabsichtigten Produktdarstellung von $f$ wird die Stetigkeit (in allen Variablen) direkt gezeigt, so daß damit $f$ als stetig erkannt wird ${ }^{7}$ ) .

Die Durchführung des Beweises erfolgt im einzelnen so:

Da die Behauptung des Satzes lokaler Natur ist, genügt es, polyzylindrische Umgebungen $U_{1} \times \ldots \times U_{n}$ eines willkürlich gewählten Punktes $\left(z_{01}, \ldots, z_{0 n}\right)$ der (offenen) Definitionsmenge der gegebenen komplexwertigen Funktion $f$ zu betrachten. Die $U_{j}$ seien dabei etwa kreisförmige Umgebungen des Punktes $z_{0 j}$ in der $z_{\text {j }}$-Ebene; sie werden so gewählt, daß $\bar{U}_{1} \times \ldots \times \bar{U}_{n}$ noch ganz in der Definitionsmenge von $f$ liegt, und daß ferner $\left|g_{j_{1} \cdots j_{\lambda}}\right|$ in $\bar{U}_{1} \times \ldots \times \bar{U}_{n}$ beschränkt und in $\bar{U}_{j_{1}} \times \ldots \times \bar{U}_{j_{\lambda}}$ sowie in allen $\bar{U}_{j_{1}} \times \ldots \times \bar{U}_{j_{\lambda}} \times \bar{U}_{k}$ im Lebesgueschen Sinn integrierbar sind. Mit den $g_{j_{1} \cdots j_{\lambda}}$ bilde man die Funktion

$$
\omega=\sum_{\lambda=1}^{n}(-1)^{\lambda} \sum_{j_{1}, \ldots, j_{\lambda}}^{*} T_{j_{1}} \ldots T_{j_{\lambda}} g_{j_{1} \ldots j_{\lambda}},
$$

wobei $T_{y}$ den durch

$$
T_{\nu} g\left(z_{1}, \ldots, z_{n}\right)=-\frac{1}{\pi} \iint_{U_{v}} \frac{g\left(\ldots, \zeta_{\nu}, \ldots\right)}{\zeta_{\nu}-z_{\nu}} d \xi_{\nu} d \eta_{\nu}
$$

definierten Operator $\left(\zeta_{v}=\xi_{v}+i \eta_{v}\right)$ und $\sum *$ Summation über voneinander verschiedene Indexkombinationen bedeuten. Die Funktion $\omega$

7 Bezüglich des Herausziehens holomorpher Faktoren aus Lösungen komplexer Differentialgleichungen bzw. komplexer Differentialungleichungen in jeweils mehreren komplexen Variablen vgl. [7] - [8]. 
löst dann das inhomogene Cauchy-Riemannsche Differentialgleichungssystem ${ }^{8}$ )

$$
\frac{\partial \omega}{\partial z_{j}^{*}}=g_{j}
$$

(vgl. A. Newlander und L. Nirenberg [4] und auch [6]-[8]).

Zunächst wird gezeigt, daß $\omega$ eine stetige Funktion von allen Variablen ist. Dazu seien $\left(z_{1}^{\prime}, \ldots, z_{n}^{\prime}\right)$ und $\left(z_{1}^{\prime \prime}, \ldots, z_{n}^{\prime \prime}\right)$ zwei fixierte Punkte des Polyzylinders $\bar{U}_{1} \times \ldots \times \bar{U}_{n}$. Dann betrachte man die Differenz der Integranden irgendeines Summandes in (1) in den betreffenden Punkten:

$$
\frac{g\left(\ldots, \zeta_{j_{v}}, \ldots, z_{i_{\mu}}^{\prime \prime}, \ldots\right)}{\left(\zeta_{j_{1}}-z_{j_{1}}^{\prime \prime}\right) \ldots\left(\zeta_{j_{\lambda}}-z_{j_{\lambda}}^{\prime \prime}\right)}-\frac{g\left(\ldots, \zeta_{j_{v}}, \ldots, z_{i_{\mu}}^{\prime}, \ldots\right)}{\left(\zeta_{j_{1}}-z_{j_{1}}^{\prime}\right) \ldots\left(\zeta_{j_{\lambda}}-z_{j_{\lambda}}^{\prime}\right)} .
$$

In diese Differenz werden nun so Summanden zwischengeschaltet, daß sich bei den zwischengeschalteten Summanden jeweils nur eine Variable $z_{x}$ ändert. Je nachdem, ob $z_{\varkappa}$ gleich einem $z_{j_{y}}(v=1, \ldots, \lambda)$ oder gleich einem $z_{k_{\mu}}(\mu=1, \ldots, n-\lambda)$ ist, haben die neuen Differenzen das Aussehen

$$
\frac{g(\ldots)}{\prod_{j_{\nu} \neq x}\left(\zeta_{j_{\nu}}-z_{j_{\nu}}\right)} \frac{z_{\varkappa}^{\prime \prime}-z_{\varkappa}^{\prime}}{\left(\zeta_{\varkappa}-z_{\varkappa}^{\prime}\right)\left(\zeta_{\varkappa}-z_{\varkappa}^{\prime \prime}\right)}
$$

bzw.

$$
\frac{1}{\Pi\left(\zeta_{j_{\nu}}-z_{j_{\nu}}\right)}\left(g\left(\ldots, z_{\varkappa}^{\prime \prime}, \ldots\right)-g\left(\ldots, z_{\varkappa}^{\prime}, \ldots\right)\right) .
$$

Für alle $z_{k}$ mit $k \neq x$ sind dabei entweder $z_{k}^{\prime}$ oder $z_{k}^{\prime \prime}$ eingesetzt $z u$ denken. Bei festgehaltenem $z_{j}^{\prime}$ konvergiere $z_{j}^{\prime \prime}(l)$ für $l \rightarrow \infty$ nach $z_{j}^{\prime}$. Benutzt man bekannte Abschätzungen von Integralen über $\bar{U}_{x}$, wobei der Integrand die Singularität

$$
\frac{1}{\left(\zeta_{x}-z_{\varkappa}^{\prime}\right)\left(\zeta_{x}-z_{x}^{\prime \prime}\right)}
$$

hat (vgl. I. N. Vekua [10]), so sieht man, daß das Integral über (2) bei $l \rightarrow \infty$ nach Null konvergiert (hierbei wird die gleichmäßige Beschränktheit von $|g|$ berücksichtigt).

Da $g$ entsprechend Voraussetzung $4^{\mathrm{O}}$ insbesondere von $z_{\varkappa}$ stetig abhängt, konvergiert (3) bei $l \rightarrow \infty$ gegen Null in jedem Punkt des

8 Anstelle von (1) kann man auch andere explizite Darstellungen der Lösung des inhomogenen Cauchy-Riemannschen Differentialgleichungssystems verwenden, falls die darin auftretenden Kerne die nachfolgenden Schlußweisen zulassen. 
Integrationsgebietes (da $g$ außer von $z_{\varkappa}$ auch von den übrigen $z_{k_{\mu}}$ abhängt, ist hierbei zu berücksichtigen, daß $g$ eine stetige Funktion aller $z_{k_{1}}, \ldots, z_{k_{n-\lambda}}$ ist). Durch Anwendung des Lebesgueschen Konvergenzsatzes folgt dann aber auch, daß das Integral bei $l \rightarrow \infty$ gegen Null konvergiert. Insgesamt ist damit gezeigt, daß jeder Summand in (1) und damit $\omega$ selbst eine stetige Funktion ist ${ }^{9}$ ).

Nun betrachte man die Funktion

$$
\Phi=w \exp (-\omega) .
$$

Unter Beachtung von Voraussetzung $1^{\mathrm{O}}$ folgt, da $\Phi$ eine stetige Funktion jeder einzelnen Variablen ist. Bei festgehaltenen übrigen Variablen ergibt sich aus Produkt- und Kettenregel (vgl. I. N. Vekua [10], Kap. I, § 10), daß

$$
\frac{\partial \Phi}{\partial z_{j}^{*}}=\left(\frac{\partial f}{\partial z_{j}^{*}}-f g_{j}\right) \exp (-\omega)=0
$$

ist (für $f \neq 0$ folgt dies unter Berücksichtigung der Definition von $g_{j}$, für $f=0$ hat man Voraussetzung $5^{0}$ zu berücksichtigen $\left.{ }^{10}\right)$ ). Aus (4) folgt nach dem Weylschen Lemma, daß $\Phi$ eine im klassischen Sinn holomorphe Funktion von jeder einzelnen Variablen $z_{j}$ ist (bei diesem Schluß ist die stetige Abhängigkeit der Funktion $\Phi$ von $z_{j}$ zu berücksichtigen $\left.{ }^{11}\right)$ ). Wendet man nun den Hartogsschen Satz an, so ergibt sich die Stetigkeit von $\Phi$ als Funktion aller Variablen. Damit ist auch

$$
f=\Phi \exp \omega
$$

als Funktion aller Variablen stetig, womit der Satz bewiesen ist.

Schlußbemerkungen:

1) Man beachte, daß die Forderung der Existenz der $g_{j_{1} \cdots j_{\lambda}}$ für $\lambda>1$ nur die Differenzierbarkeit von $(1 / w) \partial w / \partial z_{i}^{*}$, nicht die von $\partial w / \partial z_{i}^{*}$ selbst erfordert.

2) Im Fall $n=2$ wird durch Voraussetzung $4^{\mathrm{O}}$ des Satzes die Stetigkeit

\footnotetext{
${ }^{9}$ Die Ableitungen $\partial \omega / \partial z_{j}^{*}$ sind als Funktionen aller $n$ Variablen im allgemeinen nicht stetig.

$10 \mathrm{Um}$ die genannten Differentiationsregeln aus [10], Kap. I, § 10, anwenden zu können, müssen $\partial w / \partial z_{j}^{*}$ und $\partial \omega / \partial z_{j}^{*}$ (als Funktionen jeweils einer komplexen Variablen) zum $L P$ gehören. Da $\partial \omega / \partial z_{j}^{*}=g_{j}$ ist, folgt dies für $\omega$ aus der Meßbarkeit von $g_{j}$. Da andererseits $\partial f / \partial z_{j}^{*}=f g_{j}$ gilt (letzteres ist wegen $5^{\mathrm{O}}$ auch in Punkten mit $f=0$ richtig) und da $f$ (als Funktion einer Variablen) stetig ist, gehört auch $\partial f / \partial z_{j}^{*}$ zum $L^{p}$.

11 In dem in [7]-[8] betrachteten Fällen kann $\omega$ in bezüglich der $z_{j}$-Ebene isolierten Punkten unstetig sein. Da $\omega$ (und auch $w$ ) beschränkt ist, muß $\Phi$ dann aber nach dem Riemannschen Hebbarkeitssatz auch in diesen Punkten holomorph und folglich stetig sein.
} 
der $g_{i_{1} \cdots i_{\lambda}}$ (in diesem Fall von $g_{1}$ und $g_{2}$ ) von jeweils nur einer Variablen gefordert.

3) Man betrachte Lösungen $\left(w_{1}, \ldots, w_{m}\right)$ des Systems

$$
\frac{\partial w_{i}}{\partial z_{j}^{*}}=w_{i} h_{i j}\left(z_{1}, \ldots, z_{n}, w_{1}, \ldots, w_{m}\right)
$$

$(j=1, \ldots, m, i=1, \ldots, n)$, wobei die $h_{i j}$ holomorph von den $w_{v}$ abhängen sollen (sind für gewisse $w_{v}$-Werte die $h_{i j}$ betraglich durch $L$ beschränkt, so lassen sich also die rechten Seiten betraglich durch $L\left|w_{i}\right|$ abschätzen, vgl. [7]). Wegen der Holomorphie der $h_{i j}$ in den $w_{v}$ lassen sich (wie unmittelbar aus (5) folgt) auch die höheren Ableitungen der $w_{j}$ durch die $w_{v}$ selbst ausdrücken. Fordert man $h_{i j}=0$, falls $w_{i}=0$ ist, so ist

$$
g_{j}^{(i)}=\frac{1}{w_{i}} \frac{\partial w_{i}}{\partial z_{j}^{*}}=h_{i j}
$$

auch in Punkten mit $w_{i}=0$ (als Funktion jeweils einer komplexen Variablen) stetig.

Setzt man die $h_{i j}$ (bezüglich der $z_{v}$ ) genügend oft differenzierbar voraus, so genügt überhaupt die Voraussetzung, daß die $w_{i}$ von jeder einzelnen Variablen $z_{v}$ stetig abhängen sollen. Nach dem bewiesenen Satz, angewandt mit $n=2$, folgt dann, daß die $w_{i}$ und alle Ableitungen der $w_{i}$ von jeweils zwei Variablen stetig abhängen. Durch Wiederholung des Schlusses folgt schließlich, daß die $w_{i}$ von allen Variablen stetig abhängen. Das bedeutet, daß im Fall von Lösungen von Systemen der Form (5) mit in den $w_{v}$ holomorphen rechten Seiten der Satz unter der alleinigen Voraussetzung $1^{\mathrm{O}}$ gilt.

4) Will man (zum Zweck des Holomorphiebeweises von $\Phi$ ) nur beweisen, daß $\omega$ von jeweils einer Variablen $z_{j}$ stetig abhängt, so genügt es (in Abschwächung von $4^{\circ}$ ) zu wissen, daß die $g_{j_{1} \cdots j_{\lambda}}$ von jeder einzelnen Variablen $z_{k_{1}}, \ldots, z_{k_{n-\lambda}}$ stetig abhängen. Falls bekannt ist, daß die Ableitungen von $f$ selbst existieren und stetige Funktionen jeder einzelnen Variablen sind, genügt es dazu vorauszusetzen, daß $f$ als Funktion jeweils einer komplexen Variablen nur isolierte Nullstellen besitzt oder identisch verschwindet.

5) Bei dem in [5] betrachteten System

$$
\frac{\partial w}{\partial z^{*}}=\mathrm{A}_{j} w
$$

wird $g_{j}=A_{j}$ (auch für $w=0$ ) gesetzt. Entsprechend kann für Lösungen des Systems 


$$
\frac{\partial w}{\partial z_{j}^{*}}=w h_{j}\left(z_{1}, \ldots, z_{n}, w\right)
$$

im Fall $w=0$

$$
g_{j}\left(z_{1}, \ldots, z_{n}\right)=h_{j}\left(z_{1}, \ldots, z_{n}, 0\right)
$$

gesetzt werden, so daß also allgemein

$$
g_{j}\left(z_{1}, \ldots, z_{n}\right)=h_{j}\left(z_{1}, \ldots, z_{n}, w\left(z_{1}, \ldots, z_{n}\right)\right)
$$

ist. Ist $h_{j}$ holomorph in $w$, so gilt dann dieselbe Aussage wie in Bemerkung 3) auch ohne die Voraussetzung $h_{j}\left(z_{1}, \ldots, z_{n}, 0\right)=0$.

\section{Literatur}

[1] BEGEHR, H.: Eine Bemerkung zum Maximumprinzip für morphe Funktionen mehrerer komplexer Veränderlichen. - Math. Nachr. 69, 1975, 133-136.

[2] BERS, L.: Theory of pseudo-analytic functions. - [Hektographierte Vorlesungsausarbeitung.] New York University, Institute for Mathematics and Mechanics, New York, 1953.

[3] Hörmander, L.: An introduction to complex analysis in several variables. The University Series in Higher Mathematies. D. Van Nostrand Company, Inc., Princeton, New Jersey - Toronto - New York - London, 1966.

[4] Newlander, A., und L. Nirenderg: Complex analytic coordinates in almost complex manifolds. - Ann. of Math. (2) 65, 1957, 391-404.

[5] TUTSchke, W. [В. Тучке]: Теорема Хартогса для обобщенных аналитических функций многих комплексных переменных. - Dokl. Akad. Nauk SSSR 193, 1970, 765-766. Übersetzung: Hartogs' theorem for generalized analytic functions of several complex variables. - Soviet Math. Dokl. 11, 1970, 1028-1030.

[6] -»- Über die Umwandlung (auch nichtlinearer) partieller komplexer Differentialgleichungssysteme mit mehreren komplexen Variablen in Integrodifferentialgleichungssysteme und deren Lösung durch funktionalanalytische Methoden. - Math. Nachr. 58, 1973, 87-136.

[7] -»- Новое применение доказательства И. Н. Векуа к теореме Карлемана. Dokl. Akad. Nauk SSSR 214, 1974, 1276-1279. Übersetzung: A new application of I. N. Vekua's proof to Carleman's theorem. - Soviet Math. Dokl. 15, 1974, 374-378.

[8] -》- Faktorisierung von Lösungen partieller komplexer Differentialungleichungen mit Hilfe eines holomorphen und eines von Null vershiedenen Faktors. - Math. Nachr. 69, 1975, 77-83.

[9] VekuA, I. N. [И. Н. Векуа]: Об одном свойстве решений обобщенной системы уравнений Коши-Римана. - Soob̌̌č. Akad. Nauk Gruzin. SSR 14, $1953,449-453$.

[10] -»- Обобщенные аналитические функции. - Государственное издательство физико-математической литера гуры, Москва, 1959. Übersetzung: Verall- 
gemeinerte analytische Funktionen. - Mathematische Lehrbücher und Monographien II.15. Akademie-Verlag, Berlin, 1963.

[11] Beiträge zur komplexen Analysis und deren Anwendungen in der Differentialgeometrie, herausgegeben von J. NAAs. - Schriftenreihe des Zentralinstituts für Mathematik und Mechanik bei der Akademie der Wisșenschaften der DDR 18. Akademie-Verlag, Berlin, 1974.

Martin-Luther-Universität Halle-Wittenberg

Sektion Mathematik

Universitätsplatz 6

DDR 401 Halle (Saale)

Deutsche Demokratische Republik

Eingegangen am 12. Mai 1975 